\title{
ON A CONJECTURE OF GLUCK
}

\author{
JAMES P. COSSEY, ZOLTÁN HALASI, ATTILA MARÓTI, AND HUNG NGOC NGUYEN \\ Dedicated to Professor Nguyễn H. V. Hung on the occasion of his sixtieth birthday.
}

\begin{abstract}
Let $\mathbf{F}(G)$ and $b(G)$ respectively denote the Fitting subgroup and the largest degree of an irreducible complex character of a finite group $G$. A well-known conjecture of D. Gluck claims that if $G$ is solvable then $|G: \mathbf{F}(G)| \leq b(G)^{2}$. We confirm this conjecture in the case where $|\mathbf{F}(G)|$ is coprime to 6 . We also extend the problem to arbitrary finite groups and prove several results showing that the largest irreducible character degree of a finite group strongly controls the group structure.
\end{abstract}

\section{INTRODUCTION}

For a finite group $G$, let $\operatorname{Irr}(G)$ denote the set of irreducible (complex) characters of $G$, and write

$$
b(G):=\max \{\chi(1) \mid \chi \in \operatorname{Irr}(G)\},
$$

so that $b(G)$ is the largest irreducible character degree of $G$. Also, let $\mathbf{F}(G)$ denote the Fitting subgroup of $G$. In an old paper, D. Gluck [8] proved that $|G: \mathbf{F}(G)| \leq$ $b(G)^{13 / 2}$ and further conjectured that $|G: \mathbf{F}(G)| \leq b(G)^{2}$ for every solvable group $G$. Although Gluck's conjecture is still open, various partial results have been obtained by many authors. For instance, A. Espuelas [4] verified the conjecture for groups of odd order and this was extended later by S. Dolfi and E. Jabara [3] to (solvable) groups with abelian Sylow 2-subgroups. Recently, Y. Yang [31] has confirmed the conjecture for solvable 3 '-groups. The best general bound is due to A. Moretó and

Date: September 24, 2014.

2010 Mathematics Subject Classification. Primary 20C15, 20C30; Secondary 20D06.

Key words and phrases. Finite groups, solvable groups, character degrees, largest degree, Gluck's conjecture, Jordan-type theorems.

The research of the second author leading to these results has received funding from the European Union's Seventh Framework Programme (FP7/2007-2013) under grant agreement no. 318202, from ERC Limits of discrete structures Grant No. 617747 and from OTKA K84233. The research of the third author was supported by an Alexander von Humboldt Fellowship for Experienced Researchers, by the János Bolyai Research Scholarship of the Hungarian Academy of Sciences, by OTKA K84233, and by the MTA RAMKI Lendület Cryptography Research Group. The fourth author is partially supported by NSA Young Investigator Grant \#H98230-14-1-0293 and a BCAS Faculty Scholarship Award from the Buchtel College of Arts and Sciences, The University of Akron. 
T. R. Wolf in [22] where it was proved that $|G: \mathbf{F}(G)| \leq b(G)^{3}$ for every solvable group $G$.

We have seen that all the up-to-date partial results on Gluck's conjecture have been obtained with an additional condition on the order of $G$ or $G / \mathbf{F}(G)$. In this paper we establish the conjecture with a numerical restriction only on the order of $\mathbf{F}(G)$. Our first result may be considered not only as a generalization of the aforementioned theorem of Moretó and Wolf but also as an important step towards the solution of Gluck's conjecture. (As usual, $\mathbf{F}^{*}(G)$ and $\Phi(G)$ respectively denote the generalized Fitting subgroup and the Frattini subgroup of a finite group $G$.)

Theorem 1. Let $G$ be a finite $\pi$-solvable group where $\pi$ is the set of the prime divisors of $\left|\mathbf{F}^{*}(G / \Phi(G))\right|$. Then $|G: \mathbf{F}(G)| \leq b(G)^{3}$. Furthermore if $|\mathbf{F}(G / \Phi(G))|$ is not divisible by 64 nor 81 then $|G: \mathbf{F}(G)| \leq b(G)^{2}$.

Combining the second part of Theorem 1 with the results of Dolfi, Jabara and Yang mentioned above, we obtain

Corollary 2. Gluck's conjecture is true unless possibly if $|G / \mathbf{F}(G)|$ is divisible by 6 and $|\mathbf{F}(G / \Phi(G))|$ is divisible by 64 or 81 .

Proof. This follows from [3, Theorem 3], [31, Theorem 2.5], and Theorem 1.

We believe that the following result, which is a consequence of a theorem of Dolfi [2], will also be useful in a future general attack on Gluck's conjecture.

Theorem 3. Let $G$ be a finite solvable group with $G / \mathbf{F}(G)$ acting primitively on $\mathbf{F}(G) / \Phi(G)$. Then Gluck's conjecture is true for $G$.

Note that in Theorem 3 the factor group $G / \mathbf{F}(G)$ always acts completely reducibly and faithfully on $\mathbf{F}(G) / \Phi(G)$, whenever $G$ is solvable. This is a theorem of W. Gaschütz [13, III. 4.2, 4.4, 4.5].

While the problem of bounding the index of the Fitting subgroup of a finite group by its largest character degree has been studied extensively for solvable groups, not much has been done for arbitrary groups. We are aware of only one known result which is due to Gluck and is in the same paper mentioned above. That is, there exists a constant $c$ such that $|G: \mathbf{F}(G)| \leq b(G)^{c}$ for every finite group $G$. Another goal of this paper is to find an explicit polynomial bound for $|G: \mathbf{F}(G)|$ in terms of $b(G)$ for an arbitrary group $G$. Surprisingly, by using a result of R. M. Guralnick and G. R. Robinson [11] on the so-called commuting probability of finite groups, we easily deduce that this unspecified constant $c$ can be taken to be 4 .

Theorem 4. For every finite group $G$, we have $|G: \mathbf{F}(G)| \leq b(G)^{4}$.

One might ask for the best possible bounding constant $c$. We have observed that $|G| \leq b(G)^{3}$ for all non-abelian finite simple groups $G$ (see Theorem 12) and the bounding constant 3 cannot be lowered as the simple linear groups $\operatorname{SL}\left(2,2^{f}\right)$ show. 
(Recall that $\left|\mathrm{SL}\left(2,2^{f}\right)\right|=2^{f}\left(4^{f}-1\right)$ and $b\left(\mathrm{SL}\left(2,2^{f}\right)\right)=2^{f}+1$.) As we know of no finite group $G$ with $|G: \mathbf{F}(G)|>b(G)^{3}$ we put forward the following

Question 5. Is it true that $|G: \mathbf{F}(G)| \leq b(G)^{3}$ for every finite group $G$ ?

Indeed, Theorem 1 already answers Question 5 affirmatively in the case where $G$ is a finite $\pi$-solvable group where $\pi$ is the set of the prime divisors of $\left|\mathbf{F}^{*}(G / \Phi(G))\right|$. To further support this question, we answer it in the case where $G / \mathbf{F}(G)$ has no abelian composition factor.

Theorem 6. Let $G$ be a finite group. Then the product of the orders of the nonabelian composition factors of $G$ is at most $b(G)^{3}$.

Gluck's conjecture and Question 5 suggest that it would be interesting to find lower bounds for $b(G)$ in terms of indices of distinguished subgroups of $G$. Let $k(G)$ denote the number of conjugacy classes of $G$. Since $|G| / k(G) \leq b(G)^{2}$, we see that an upper bound for $k(G)$ provides the corresponding lower bound for $b(G)$ (see Section 7). In fact, the problem of finding upper bounds for $k(G)$ in terms of distinguished subgroups of $G$ has been studied considerably in the literature. One of the notable results, motivated by a question of J. G. Thompson on bounding $k(G)$ by the order of a so-called nilpotent injector of (the solvable group) $G$, is due to Robinson [26] who proved (among other results) that there is a well determined function $f$ such that $k(G) \leq f(|\mathbf{F}(G)|)$ for every solvable group $G$. We exploit Robinson's ideas (see also [11, Theorem 13 (ii)]) to obtain the following theorem whose consequence can be viewed as a weak form of Gluck's conjecture.

Theorem 7. Let $G$ be a finite $\pi$-solvable group where $\pi$ is the set of the prime divisors of $\left|\mathbf{F}^{*}(G)\right|$. Then $k(G) \leq|G|_{\pi}$ and consequently, if $H$ is a Hall $\pi$-subgroup of $G$ then $|G: H| \leq b(G)^{2}$. In particular, if $G$ is a solvable group and $H$ is a Hall $\pi$-subgroup of $G$ where $\pi$ is the set of the prime divisors of $|\mathbf{F}(G)|$, then $|G: H| \leq b(G)^{2}$.

I. M. Isaacs and D. S. Passman [16] proved a Jordan-type theorem which says that there exists a real-valued function $f(x)$ so that every finite group $G$ has an abelian subnormal subgroup of index at most $f(b(G)$ ) (and at least $b(G)$ ). As a consequence of Theorem 4, we show that $f(x)$ can be taken to be $x^{8}$. We combine this with a result of M. W. Liebeck and L. Pyber [20, Theorem 3] on bounding the class number of a finite group in terms of the order of a solvable subgroup to obtain

Theorem 8. Every finite group $G$ contains an abelian subnormal subgroup of index at most $b(G)^{8}$ and a solvable subgroup of index at most $b(G)^{2}$.

Since this paper was written L. Pyber informed us that in fact Gluck [8] has shown that there exists a universal constant $K$ so that in any finite group $G$ there exists an abelian subgroup of index at most $b(G)^{K}$. Pyber also pointed on a result of A. Chermak and A. Delgado [15, Theorem 1.41] saying that if a finite group contains 
an abelian subgroup of index $n$ then it also contains a characteristic abelian subgroup of index at most $n^{2}$. Using this result and the proof of Theorem 8, we see that every finite group $G$ contains a characteristic abelian subgroup of index at most $b(G)^{12}$. This and our Question 5 motivated Pyber [25] to ask the following question. Is it true that for a finite group $G$ there exists an abelian normal subgroup of index at most $b(G)^{3}$ ?

The paper is organized as follows. In Section 2 we introduce bases and state two background results. In Section 3 we translate some information about bases to 'large' orbits. Theorems 1 and 3 are proved in Section 4. In Section 5, we prove that every non-abelian finite simple group $S$ has an irreducible character that is extendible to $\operatorname{Aut}(S)$ of degree at least $|S|^{1 / 3}$ - a crucial result that will be used in Section 6 to prove Theorem 6. Finally, Theorems 4, 7, and 8 are proved in Section 7.

\section{BASES}

The notion of a base is fundamental in permutation group theory and also in computational group theory. For a finite permutation group $H \leq \operatorname{Sym}(\Omega)$, a subset of the finite set $\Omega$ is called a base for $H$ if its pointwise stabilizer in $H$ is the identity. A base of minimal size is called a minimal base.

There are a number of results on minimal base sizes of linear groups. One of these is the following.

Theorem 9 ([12], Theorem 1.1). Let $V$ be a finite vector space over a field of characteristic $p$. If $G \leq \mathrm{GL}(V)$ is a p-solvable group which acts irreducibly on $V$, then the minimal base size for $G$ is at most 2 unless $p=2$ or 3 , when the minimal base size for $G$ is at most 3 .

Let us remark that Theorem 9 is best possible. There are infinitely many solvable linear groups $G \leq \mathrm{GL}(V)$ acting irreducibly on $V$ with $|G|>|V|^{2}$ for $p=2$ or 3 (see [24, Theorem 1] or [30, page 1097]).

To state the most general form of the next theorem we introduce yet another definition. Two bases $B_{1}$ and $B_{2}$ for $H \leq \mathrm{GL}(V)$ are said to be non-equivalent if there is no $h \in H$ with $B_{1}^{h}=B_{2}$. The next result concerns such bases in the case where $H$ is a solvable primitive linear group.

Theorem 10 (Dolfi [2], Theorem 3.4). Let $G$ be a solvable primitive subgroup of $\mathrm{GL}(V)=\mathrm{GL}(n, q)$ where $V$ is a vector space of dimension $n$ over a field of order $q$ and characteristic $p$. Then $G$ has at least p pairwise non-equivalent bases each of size 2 unless one of the following cases holds for $G$.

(1) $\mathrm{GL}(2,2)$;

(2) $\mathrm{SL}(2,3)$ or $\mathrm{GL}(2,3)$;

(3) $3^{1+2} \cdot \mathrm{SL}(2,3)$ or $3^{1+2} \cdot \mathrm{GL}(2,3) \leq \mathrm{GL}(6,2)$; 
(4) $\left(Q_{8} \circ Q_{8}\right) H \leq \mathrm{GL}(4,3)$ where $H$ is isomorphic to a subgroup of index 1 , 2, or 4 of $\mathrm{O}^{+}(4,2)$.

\section{LARGE ORBITS}

In this section we translate the results in the previous section to results about 'large' orbits. By a 'large' orbit of a finite group $G$ on a vector space $V$ (possibly of mixed characteristic) we mean an orbit of length at least $|G|^{1 / 2}$ or $|G|^{1 / 3}$. The existence of such an orbit is equivalent to the existence of a vector $v \in V$ with the property that $\left|\mathbf{C}_{G}(v)\right| \leq|G|^{1 / 2}$ or $\left|\mathbf{C}_{G}(v)\right| \leq|G|^{2 / 3}$. This occurs for example if $G$ admits a base of size at most 2 or 3 on $V$. Our method below is part of Gluck's strategy [8] to produce an irreducible character of large degree (for this see Section 4).

Lemma 11. Let $G$ be a finite group acting completely reducibly and faithfully on a finite module $V$, possibly of mixed characteristic. Suppose that $G$ is $\pi$-solvable where $\pi$ is the set of prime divisors of $|V|$. Then we have the following.

(1) There exists a vector $v$ in $V$ such that $\left|\mathbf{C}_{G}(v)\right| \leq|G|^{2 / 3}$.

(2) If $|V|$ is not divisible by 64 nor 81 then there exists a vector $v$ in $V$ such that $\left|\mathbf{C}_{G}(v)\right| \leq|G|^{1 / 2}$.

(3) If $V$ is a primitive $G$-module and $G$ is solvable, then there exists a vector $v$ in $V$ such that $\left|\mathbf{C}_{G}(v)\right| \leq|G|^{1 / 2}$ unless $|V|=3^{4}$ and $G$ is a unique subgroup (up to conjugacy) in $G L(V)$ of order 1152.

Proof. Let us first prove parts (1) and (2) of the lemma. We use induction on the size of $V$. (If $|V|$ is a prime then the result is clear.)

Suppose that $V$ is reducible. Put $\epsilon=1 / 2$ if $|V|$ is not divisible by 64 nor 81 and $2 / 3$ otherwise. We have $V=U \oplus W$ where $U$ and $W$ are non-zero $G$-modules of possibly mixed characteristic. Then $G / N$ acts faithfully and completely reducibly on $U$, a module of size smaller than $|V|$, where $N=\mathbf{C}_{G}(U)$. So by the induction hypothesis, there exists a vector $u$ in $U$ with $\left|\mathbf{C}_{G / N}(u)\right| \leq|G / N|^{\epsilon}$. Note that $N$ acts faithfully and completely reducibly on $W$ and thus by induction, there exists a vector $w$ in $W$ with $\left|\mathbf{C}_{N}(w)\right| \leq|N|^{\epsilon}$. Put $v=u+w$. Then $\mathbf{C}_{G}(v) N / N \leq \mathbf{C}_{G / N}(u)$ and so

$$
\left|\mathbf{C}_{G}(v): N \cap \mathbf{C}_{G}(v)\right| \leq|G / N|^{\epsilon} .
$$

But

These yield

$$
\left|N \cap \mathbf{C}_{G}(v)\right|=\left|\mathbf{C}_{N}(v)\right|=\left|\mathbf{C}_{N}(w)\right| \leq|N|^{\epsilon}
$$

$$
\left|\mathbf{C}_{G}(v)\right| \leq|G|^{\epsilon} .
$$

Thus we may assume that $V$ is irreducible of characteristic $p$. Then by Theorem 9 there exists a base of size at most 3 and, in case $p \geq 5$, a base of size at most 2 . In particular there exists a vector $v$ in $V$ with the property that $\left|\mathbf{C}_{G}(v)\right| \leq|G|^{2 / 3}$ and a vector $v$ in $V$ with the property that $\left|\mathbf{C}_{G}(v)\right| \leq|G|^{1 / 2}$, in case $p \geq 5$. This proves 
part (1) and also part (2) of the lemma in case $p \geq 5$. Suppose that the conditions of part (2) hold with $p=2$ or 3 . Then $|V| \leq 32$ or $|V| \leq 27$ in the respective cases. But in these cases one can check the validity of the lemma by GAP [7].

Let us now turn to the proof of part (3) of the lemma. Suppose that $V$ is a primitive $G$-module and that $G$ is a solvable group. We may also assume that the minimal base size for $G$ on $V$ is at least 3. By Theorem 10 this happens only if case (3) or (4) of Theorem 10 holds. But in these cases one can check by GAP [7] that there always exists a vector $v$ in $V$ with $\left|\mathbf{C}_{G}(v)\right| \leq|G|^{1 / 2}$, except when $|V|=3^{4}$ and $G$ is a unique subgroup (up to conjugacy) in $G L(V)$ of order 1152.

Remark. Notice that $G=\operatorname{GL}(2,2)$ \ $\operatorname{Sym}(3)$ and $\operatorname{GL}(2,3)$ \ $\operatorname{Sym}(2)$ are imprimitive linear groups acting on a vector space $V$ of size 64 and 81 respectively with the property that there does not exist a vector $v \in V$ with $\left|\mathbf{C}_{G}(v)\right| \leq|G|^{1 / 2}$. Therefore the second and third statements of Lemma 11 are best possible in some sense.

\section{Proofs of Theorems 1 And 3}

Let $G$ be a finite $\pi$-solvable group where $\pi$ is the set of the prime divisors of $\left|\mathbf{F}^{*}(G / \Phi(G))\right|$. Then $\mathbf{F}^{*}(G / \Phi(G))=\mathbf{F}(G / \Phi(G))$ and so $\mathbf{F}(G / \Phi(G))=\mathbf{F}(G) / \Phi(G)$ is a faithful $G / \mathbf{F}(G)$-module. By Gaschütz's theorem (see [13, III. 4.5]) we also have that $\mathbf{F}(G) / \Phi(G)$ is a completely reducible $G / \mathbf{F}(G)$-module. Therefore $\operatorname{Irr}(\mathbf{F}(G) / \Phi(G))$ is a completely reducible and faithful $G / \mathbf{F}(G)$-module as well. By applying Lemma 11, we have an irreducible character $\lambda$ of $\mathbf{F}(G) / \Phi(G)$ (and thus of $\mathbf{F}(G)$ ) whose stabilizer in $G / \mathbf{F}(G)$ has size at most $|G: \mathbf{F}(G)|^{a}$ where $a=1 / 2$ if the conditions of the second or third statements of Lemma 11 are satisfied and $a=2 / 3$ otherwise. This means that $\lambda$ lies in a $G$-orbit of size at least $|G: \mathbf{F}(G)|^{1-a}$. Theorem 1 now follows by Clifford's theorem.

Now we turn to the proof of Theorem 3. Let $G$ be a finite solvable group with $G / \mathbf{F}(G)$ acting faithfully and primitively on $\mathbf{F}(G) / \Phi(G)$. Then $G / \mathbf{F}(G)$ also acts faithfully and primitively on $\operatorname{Irr}(\mathbf{F}(G) / \Phi(G))$. By the previous paragraph we arrive to a conclusion unless the $G / \mathbf{F}(G)$-module $\operatorname{Irr}(\mathbf{F}(G) / \Phi(G))$ has size $3^{4}$ and $|G / \mathbf{F}(G)|=$ 1152. This happens only if the faithful, primitive $G / \mathbf{F}(G)$-module $V=\mathbf{F}(G) / \Phi(G)$ has size $3^{4}$.

As mentioned in the introduction, for a finite group $T$ let $k(T)$ be the number of conjugacy classes of $T$. The group $T=G / \Phi(G)$ has the form $H V$, a split extension of $H$ and $V$, where $H$ is isomorphic to $G / \mathbf{F}(G)$ and it acts the same way on $V$ as $G / \mathbf{F}(G)$ does (apply [13, III. 4.4] to the group $T$ with a trivial Frattini subgroup). Thus $k(G / \Phi(G))=k(H V)$. But by GAP [7] we have $k(H V) \leq|V|$ which gives $k(G / \Phi(G)) \leq|\mathbf{F}(G) / \Phi(G)|$. This and Nagao's result [23] then gives

$$
k(G) \leq k(G / \Phi(G)) k(\Phi(G)) \leq|\mathbf{F}(G)| .
$$

But then the inequality $|G| / k(G) \leq b(G)^{2}$ yields the desired conclusion. 


\section{Extendible CharaCters of LARGE DEGREe in Simple Groups}

Our proof of Theorem 6 relies on the classification of finite simple groups. The specific consequence of the classification is the following result, which may have other applications.

Theorem 12. Let $S$ be a finite non-abelian simple group and let $d(S)$ denote the largest degree of an irreducible character of $S$ that extends to $\operatorname{Aut}(S)$. Then we have $d(S) \geq|S|^{1 / 3}$.

The proof of Theorem 12 is fairly straightforward for sporadic simple groups and simple groups of Lie type. So most of this section is devoted to the proof for the alternating groups. We first recall some basic combinatorics connecting partitions, Young diagrams, and representation theory of the alternating and symmetric groups.

Let $n$ be a natural number. It is well-known that there are bijective correspondences between the partitions of $n$, the Young diagrams of cardinality $n$, and the irreducible complex characters of $\mathrm{S}_{n}$. Let $\lambda$ be a partition of $n$. That is, $\lambda$ is a finite sequence $\left(\lambda_{1}, \lambda_{2}, \ldots, \lambda_{k}\right)$ for some $k$ such that $\lambda_{1} \geq \lambda_{2} \geq \cdots \geq \lambda_{k}$ and $\lambda_{1}+\lambda_{2}+\cdots+\lambda_{k}=n$. Each $\lambda_{i}$ is called a part of $\lambda$.

The Young diagram corresponding to $\lambda$, denoted by $Y_{\lambda}$, is defined to be the finite subset of $\mathbb{N} \times \mathbb{N}$ such that

$$
(i, j) \in Y_{\lambda} \text { if and only if } i \leq \lambda_{j}
$$

Two partitions of $n$ whose associated Young diagrams transform into each other when reflected about the line $y=x$ are called conjugate partitions. The partition conjugate to $\lambda$ is denoted by $\bar{\lambda}$. If $\lambda=\bar{\lambda}$ then $Y_{\lambda}$ is symmetric and we say that $\lambda$ is self-conjugate. For each node $(i, j) \in Y_{\lambda}$, we define the so-called hook length $h(i, j)$ to be the number of nodes that are directly above it, directly to the right of it, or equal to it. More precisely,

$$
h(i, j):=1+\lambda_{j}+\bar{\lambda}_{i}-i-j .
$$

We denote by $\chi_{\lambda}$ or $\chi_{Y_{\lambda}}$ the irreducible character of $\mathrm{S}_{n}$ corresponding to $\lambda$ and $Y_{\lambda}$. The degree of $\chi_{\lambda}$ is given by the hook-length formula of J.S. Frame, G. B. Robinson, and R. M. Thrall, see [6]:

$$
f_{\lambda}:=\chi_{\lambda}(1)=\chi_{Y_{\lambda}}(1)=\frac{n !}{\prod_{(i, j) \in Y_{\lambda}} h(i, j)} .
$$

The irreducible characters of $A_{n}$ can be obtained by restricting those of $S_{n}$ to $A_{n}$. More explicitly, $\chi_{\lambda} \downarrow_{\mathrm{A}_{n}}=\chi_{\bar{\lambda}} \downarrow_{\mathrm{A}_{n}}$ is irreducible if $\lambda$ is not self-conjugate. Otherwise, $\chi_{\lambda} \downarrow_{A_{n}}$ splits into two different irreducible characters of the same degree. In short, the degrees of the irreducible characters of $\mathrm{A}_{n}$ are labelled by partitions of $n$ and are 
given by

$$
\widetilde{f}_{\lambda}= \begin{cases}f_{\lambda} & \text { if } \lambda \neq \bar{\lambda} \\ f_{\lambda} / 2 & \text { if } \lambda=\bar{\lambda}\end{cases}
$$

Given a partition $\lambda$ of $n$, we define $A(\lambda)$ and $R(\lambda)$ to be the sets of nodes that can be respectively added or removed from $Y_{\lambda}$ to obtain another Young diagram corresponding to a certain partition of $n+1$ or $n-1$ respectively. It is known (see $[19, \S 2]$ for instance) that

$$
|A(\lambda)|<\sqrt{2 n}+1 \text { and }|R(\lambda)|<\sqrt{2 n} .
$$

The branching rule $[17, \oint 9.2]$ asserts that the restriction of $\chi_{\lambda}$ to $S_{n-1}$ is a sum of irreducible characters $\chi_{Y_{\lambda} \backslash\{(i, j)\}}$ as $(i, j)$ goes over all nodes in $R(\lambda)$. Also, by Frobenius reciprocity, the induction of $\chi_{\lambda}$ to $S_{n+1}$ is a sum of irreducible characters $\chi_{Y_{\lambda} \cup\{(i, j)\}}$ as $(i, j)$ goes over all nodes in $A(\lambda)$.

Lemma 13. Theorem 12 is true for the simple alternating groups.

Proof. The validity of the lemma can be checked by computer for all values of $n$ in the range $5 \leq n \leq 30$. We will prove by induction on $n \geq 30$ that $d\left(\mathrm{~A}_{n+1}\right) \geq$ $(n+1)^{1 / 3} d\left(\mathrm{~A}_{n}\right)$. This then implies the inequality $d\left(\mathrm{~A}_{n}\right) \geq(n ! / 2)^{1 / 3}$ immediately.

Let $\theta$ be an irreducible character of $\mathrm{A}_{n}$ with $n \geq 30$ such that $\theta$ is extendible to $\mathrm{S}_{n}$ and $\theta(1)=d\left(\mathrm{~A}_{n}\right)$. Assume that $\theta$ extends to $\chi \in \operatorname{Irr}\left(\mathbf{S}_{n}\right)$. Let $\lambda$ and $Y$ be respectively the partition and Young diagram corresponding to $\chi$. Consider the induction of $\chi$ to $\mathrm{S}_{n+1}$. We have

$$
\chi^{\mathrm{S}_{n+1}}(1)=(n+1) \chi(1)=(n+1) d\left(\mathrm{~A}_{n}\right)
$$

and

$$
\chi^{\mathrm{S}_{n+1}}=\sum_{(i, j) \in A(\lambda)} \chi_{Y \cup\{(i, j)\}}
$$

by the branching rule. There are two cases arising.

(1) None of the Young diagrams in $\{Y \cup\{(i, j)\} \mid(i, j) \in A(\lambda)\}$ are symmetric. Then the irreducible characters $\chi_{Y \cup\{(i, j)\}}$ restrict irreducibly to $\mathrm{A}_{n+1}$ so that

$$
\chi_{Y \cup\{(i, j)\}}(1) \leq d\left(\mathrm{~A}_{n+1}\right)
$$

for every $(i, j) \in A(\lambda)$. We therefore deduce that

$$
\chi^{\mathrm{S}_{n+1}}(1) \leq|A(\lambda)| d\left(\mathrm{~A}_{n+1}\right) .
$$

Since $|A(\lambda)|<\sqrt{2 n}+1$ and $\chi^{\mathrm{S}_{n+1}}(1)=(n+1) d\left(\mathrm{~A}_{n}\right)$, it follows that

$$
(n+1) d\left(\mathrm{~A}_{n}\right) \leq\lceil\sqrt{2 n}\rceil d\left(\mathrm{~A}_{n+1}\right)
$$


where $\lceil x\rceil$ denotes the smallest integer not smaller than $x$. Thus as $n \geq 30$ we conclude that

$$
d\left(\mathrm{~A}_{n+1}\right) \geq \frac{n+1}{\lceil\sqrt{2 n}\rceil} d\left(\mathrm{~A}_{n}\right) \geq(n+1)^{1 / 3} d\left(\mathrm{~A}_{n}\right),
$$

as desired.

(2) There is a symmetric Young diagram in $\{Y \cup\{(i, j)\} \mid(i, j) \in A(\lambda)\}$. Then there is exactly one such diagram and at most $\lceil\sqrt{2 n}-1\rceil$ non-symmetric diagrams in $\{Y \cup\{(i, j)\} \mid(i, j) \in A(\lambda)\}$. Let $Y^{\prime}$ be that symmetric Young diagram and $\mu$ be the corresponding partition. By the branching rule, we have

$$
\chi_{Y^{\prime}} \downarrow s_{n}=\sum_{(i, j) \in R(\mu)} \chi_{Y^{\prime} \backslash\{(i, j)\}} .
$$

Now there are two subcases arising.

(a) None of the Young diagrams of the form $Y^{\prime} \backslash\{(i, j)\}$ where $(i, j) \in R(\mu)$ are symmetric. Then the characters associated to these diagrams restrict irreducibly to $\mathrm{A}_{n}$ and thus their degrees are at most $d\left(\mathrm{~A}_{n}\right)$. As $|R(\mu)|<\sqrt{2 n+2}$, we deduce that

$$
\chi_{Y^{\prime}}(1)=\sum_{(i, j) \in R(\mu)} \chi_{Y^{\prime} \backslash\{(i, j)\}}(1) \leq\lceil\sqrt{2 n+2}-1\rceil d\left(\mathrm{~A}_{n}\right) .
$$

Combining (5.1), (5.2), and the last inequality, we have

$$
\begin{aligned}
(n+1) d\left(\mathrm{~A}_{n}\right) & =\sum_{(i, j) \in A(\lambda)} \chi_{Y \cup\{(i, j)\}}(1) \\
& =\chi_{Y^{\prime}}(1)+\sum_{(i, j) \in A(\lambda), Y \cup\{(i, j)\} \neq Y^{\prime}} \chi_{Y \cup\{(i, j)\}}(1) \\
& \leq\lceil\sqrt{2 n+2}-1\rceil d\left(\mathrm{~A}_{n}\right)+(|\mathrm{A}(\lambda)|-1) d\left(\mathrm{~A}_{n+1}\right) \\
& \leq\lceil\sqrt{2 n+2}-1\rceil d\left(\mathrm{~A}_{n}\right)+\lceil\sqrt{2 n}-1\rceil d\left(\mathrm{~A}_{n+1}\right) .
\end{aligned}
$$

Thus as $n \geq 30$ we deduce that

$$
d\left(\mathrm{~A}_{n+1}\right) \geq \frac{n+2-\lceil\sqrt{2 n+2}\rceil}{\lceil\sqrt{2 n}-1\rceil} d\left(\mathrm{~A}_{n}\right) \geq(n+1)^{1 / 3} d\left(\mathrm{~A}_{n}\right),
$$

as wanted.

(b) There is a symmetric Young diagram in $\left\{Y^{\prime} \backslash\{(i, j)\} \mid(i, j) \in R(\mu)\right\}$. Then there is exactly one such diagram and at most $\lceil\sqrt{2 n+2}-2\rceil$ non-symmetric diagrams in $\left\{Y^{\prime} \backslash\{(i, j)\} \mid(i, j) \in R(\mu)\right\}$. Let $Y^{\prime \prime}$ be that symmetric Young diagram and $\nu$ be the corresponding partition. So we have two symmetric Young diagrams $Y^{\prime}$ and $Y^{\prime \prime}$ and $Y^{\prime \prime}$ is obtained from $Y^{\prime}$ by removing a node. Therefore, if another node is 
removed from $Y^{\prime \prime}$ to get a Young diagram (of size $n-1$ ), the resulting diagram can not be symmetric. Therefore, again by the branching rule,

$$
\chi_{Y^{\prime \prime}}(1) \leq\lceil\sqrt{2 n}-1\rceil d\left(\mathrm{~A}_{n-1}\right) .
$$

It follows that

$$
\chi_{Y^{\prime}}(1) \leq\lceil\sqrt{2 n}-1\rceil d\left(\mathrm{~A}_{n-1}\right)+\lceil\sqrt{2 n+2}-2\rceil d\left(\mathrm{~A}_{n}\right) .
$$

Therefore, as in subcase (a),

$$
\begin{aligned}
(n+1) d\left(\mathrm{~A}_{n}\right) & =\chi_{Y^{\prime}}(1)+\sum_{(i, j) \in A(\lambda), Y \cup\{(i, j)\} \neq Y^{\prime}} \chi_{Y \cup\{(i, j)\}}(1) \\
& \leq\lceil\sqrt{2 n}-1\rceil d\left(\mathrm{~A}_{n-1}\right)+\lceil\sqrt{2 n+2}-2\rceil d\left(\mathrm{~A}_{n}\right)+\lceil\sqrt{2 n}-1\rceil d\left(\mathrm{~A}_{n+1}\right) .
\end{aligned}
$$

Using the induction hypothesis that $d\left(\mathrm{~A}_{n-1}\right) \leq n^{-1 / 3} d\left(\mathrm{~A}_{n}\right)$ and noting $n \geq 30$, we obtain

$$
d\left(\mathrm{~A}_{n+1}\right) \geq \frac{n+3-\lceil\sqrt{2 n+2}\rceil-\lceil\sqrt{2 n}-1\rceil n^{-1 / 3}}{\lceil\sqrt{2 n}-1\rceil} d\left(\mathrm{~A}_{n}\right) \geq(n+1)^{1 / 3} d\left(\mathrm{~A}_{n}\right),
$$

as wanted. The proof is complete.

Remark. It can be shown by viewing Young diagrams of rectangular shapes that for any $\delta>0$ there exists an $N>0$ such that whenever $n>N$ we have $d\left(\mathrm{~A}_{n}\right)>n !^{(1 / 2)-\delta}$.

Proof of Theorem 12. If $S$ is a simple sporadic group or the Tits group, the proof is a routine check from the Atlas [1]. As the alternating groups have been already handled in Lemma 13, we can assume that $S$ is a simple group of Lie type in characteristic $p$. We then realize that $S$ has the so-called Steinberg character $\operatorname{St}_{S}$ of degree $\mathrm{St}_{S}(1)=$ $|S|_{p}$, the $p$-part of the order of $S$, and $\mathrm{St}_{S}$ is extendible to $\operatorname{Aut}(S)$ (see [5]). Now we can check the inequality $|S|_{p}>|S|^{1 / 3}$ easily by consulting the list of simple groups and their orders, see [1, p. xvi] for instance.

Remark. The bounding constant $1 / 3$ in Theorem 12 is tight since the order of $\operatorname{PSL}_{2}(q)$ is $q\left(q^{2}-1\right) / \operatorname{gcd}(2, q-1)$ and the largest degree of an irreducible character of $\operatorname{PSL}_{2}(q)$ extendible to $\operatorname{Aut}\left(\mathrm{PSL}_{2}(q)\right)$ is either $q$ or $q+1$.

\section{Bounding THE PRODUCT OF THE ORDERS OF NON-ABELIAN COMPOSITION FACTORS}

In this section we prove Theorem 6 . To do that, we first apply the main result of the previous section to obtain the following.

Proposition 14. Let $N$ be a non-abelian minimal normal subgroup of a finite group G. Then

$$
b(G)^{3} \geq b(G / N)^{3}|N|
$$


Proof. Since $N$ is a non-abelian minimal normal subgroup of the finite group $G$, we know that $N \cong S \times \cdots \times S$, a direct product of say $k$ copies of a non-abelian simple group $S$. By Theorem 12, we know that there exists a non-principal character $\alpha \in$ $\operatorname{Irr}(S)$ such that $\alpha$ extends to $\operatorname{Aut}(S)$ and $\alpha(1) \geq|S|^{1 / 3}$. In particular, the product character $\psi:=\alpha \times \cdots \times \alpha$ is invariant under $\operatorname{Aut}(N)=\operatorname{Aut}(S)$ < $\mathbf{S}_{k}$. By [21], the character $\psi$ extends to $\operatorname{Aut}(N)$. Since $N$ is embedded into $G / \mathbf{C}_{G}(N)$ and $G / \mathbf{C}_{G}(N)$ embeds into $\operatorname{Aut}(N)$, the character $\psi$ extends to $G / \mathbf{C}_{G}(N)$ and therefore $\psi$ extends to a character $\chi$ of $G$.

Applying Gallagher's Theorem [14, Corollary 6.17], we deduce that there is a bijection $\lambda \mapsto \lambda \chi$ between $\operatorname{Irr}(G / N)$ and the set of irreducible characters of $G$ lying above $\psi$. In particular, by taking $\lambda$ to be an irreducible character of largest degree, we deduce that $b(G / N) \chi(1)=b(G / N) \psi(1)$ is a character degree of $G$. It follows that

$$
b(G) \geq b(G / N) \psi(1) .
$$

Recall that $\psi:=\alpha \times \cdots \times \alpha$ and $\alpha(1) \geq|S|^{1 / 3}$. Thus $\psi(1) \geq|N|^{1 / 3}$. It follows by the above inequality that

$$
b(G) \geq b(G / N)|N|^{1 / 3},
$$

and this completes the proof.

Theorem 6 now is a consequence of Proposition 14.

Proof of Theorem 6. In light of Proposition 14, we see that if $N$ is a minimal normal subgroup of a finite group $X$, then

$$
\begin{gathered}
b(X) \geq b(X / N) \text { if } N \text { is abelian, and } \\
b(X) \geq b(X / N)|N|^{1 / 3} \text { if } N \text { is non-abelian. }
\end{gathered}
$$

Let $1=M_{0}<M_{1}<\cdots<M_{k}=G$ be a chief series of $G$. That is, $M_{i+1} / M_{i}$ is a minimal normal subgroup of $G / M_{i}$ for every $i=0,1, \ldots, k-1$. Arguing by induction on the number of chief factors of $G$, we deduce that the product of the orders of all non-abelian chief factors of $G$ is at most $b(G)^{3}$, which also means that the product of the orders of all non-abelian composition factors of $G$ is at most $b(G)^{3}$. The proof is complete.

\section{Distinguished Subgroups And THE LARGEST CHARACTER DEGREE}

We need two preliminary lemmas to prove Theorem 7.

Lemma 15. Let $G$ be a finite $p$-solvable group and $V$ an elementary abelian normal $p$-subgroup of $G$ for some prime $p$. Suppose that $G / V$ acts faithfully on $V$. Then $k(G) \leq|G|_{p}$. 
Proof. The hypothesis implies that $\mathbf{O}_{p^{\prime}}(G)=1$. But then Theorem 2.6(a) of [28] states that $G$ has a unique $p$-block, the principal block. The defect group of the principal block is a Sylow $p$-subgroup of $G$. Hence by the $p$-solvable case of Brauer's $k(B)$-problem (see [9]) we obtain $k(G) \leq|G|_{p}$.

Lemma 16. Let $G$ be a $\pi$-solvable finite group, where $\pi$ is the set of prime divisors of $\left|\mathbf{F}^{*}(G)\right|$. Then $\mathbf{F}^{*}(G / \Phi(G))=\mathbf{F}^{*}(G) / \Phi(G)$. In particular, the set of prime divisors of $\left|\mathbf{F}^{*}(G / \Phi(G))\right|$ is a subset of $\pi$.

Proof. Let $\bar{G}=G / \Phi(G)$ and let $N$ be the inverse image of $\mathbf{F}^{*}(\bar{G})$ in $G$, that is, $N / \Phi(G)=\mathbf{F}^{*}(G / \Phi(G))$. Then $\mathbf{F}^{*}(\bar{G})=E(\bar{G}) \circ \mathbf{F}(\bar{G})$, the central product of $E(\bar{G})$, the subgroup generated by all the quasisimple subnormal subgroups of $\bar{G}$ and $\mathbf{F}(\bar{G})$, the Fitting subgroup of $\bar{G}$. Moreover, $E(\bar{G})$ is itself a central product of subnormal quasisimple subgroups of $\bar{G}$. Here $\mathbf{Z}(E(\bar{G})) \leq \mathbf{F}(\bar{G})$. As $\mathbf{F}(\bar{G})=\mathbf{F}(G) / \Phi(G)$, we have that $N / \mathbf{F}(G)$ is a direct product of some non-abelian simple groups. (Unless, $N=\mathbf{F}(G)$, in which case we are done.) Since $N / \mathbf{F}(G)$ is a $\pi$-solvable group, we have that it is a $\pi^{\prime}$-group. As $\mathbf{F}(G)$ is a $\pi$-group, $(|\mathbf{F}(G)|,|N: \mathbf{F}(G)|)=1$, so the Schur-Zassenhaus theorem can be applied. We get $N=\mathbf{F}(G) \rtimes K$ for some $\pi^{\prime}$-group $K \leq G$.

Now, we prove that we can assume that $\Phi(\mathbf{F}(G))=1$. Assuming the converse, let $\tau: G \rightarrow G / \Phi(\mathbf{F}(G))$ be the natural homomorphism. Then $\Phi(\mathbf{F}(G)) \leq \Phi(G)$, so $\Phi(\tau(G))=\tau(\Phi(G))$ holds. Furthermore, $\tau(\mathbf{F}(G))=\mathbf{F}(\tau(G))$, and $\mathbf{F}^{*}(\tau(G)) \leq \tau(N)$ also follows.

We claim that $K$ acts faithfully on $\tau(\mathbf{F}(G))$. Indeed, let $L \leq K$ be the kernel of this action and let $P$ be the Sylow $p$-subgroup of $\mathbf{F}(G)$ for some $p \in \pi$. Then $L$ acts trivially on the vector space $P / \Phi(P) \simeq \tau(P)$. Now, we use the idea of the proof of [13, III. Satz 3.17], which we recall here only for completeness. Let $\left\{a_{1}, \ldots, a_{l}\right\}$ be a basis of $P / \Phi(P)$. Then the set $X=\left\{\left(a_{1} t_{1}, \ldots, a_{l} t_{l}\right) \mid t_{1}, \ldots, t_{l} \in \Phi(P)\right\}$ is fixed by $L$, and $L / C_{L}(P)$ acts semiregularly on $X$, since every element of $X$ provides a (minimal) generating set of $P$. It follows that $\left.\left|L / C_{L}(P)\right||| X|=| \Phi(P)\right|^{l}$. But $L$ is a $p^{\prime}$-group, while $\Phi(P)$ is a $p$-group. So $L / C_{L}(P)=1$. As this holds for every Sylow subgroup of $\mathbf{F}(G)$ we get $L \leq C_{K}(\mathbf{F}(G))=1$.

As $E(\tau(G)) \leq \tau(K)$ centralizes $\mathbf{F}(\tau(G))$, we get $E(\tau(G))=1$, so $\mathbf{F}^{*}(\tau(G))=$ $\mathbf{F}(\tau(G))=\tau(\mathbf{F}(G))$ is a $\pi$-group and we can use an induction argument on the order of the finite group.

Now, let $\Phi(\mathbf{F}(G))=1$. Then $\mathbf{F}(G)$ is a direct product of elementary abelian groups, and it is completely reducible as a $K$-module by Maschke's theorem. As $\mathbf{F}^{*}(\bar{G})=K \times \mathbf{F}(\bar{G})$, we get $\mathbf{F}(G)=\Phi(G) \oplus U$ as a $K$-module, where $U$ is centralized by $K$ and $K$ acts faithfully on $\Phi(G)$ by conjugation.

Let now $M=\mathbf{N}_{G}(K) \geq U K$. We claim that $G=M \Phi(G)$. Notice that this is sufficient to prove the lemma, since $G=M$ implies that $K$ is normal in $G$ and so $K \leq \mathbf{F}^{*}(G)$. 
To prove that $G=M \Phi(G)$, let $g \in G$ be arbitrary. Then $K^{g} \leq N \triangleleft G$, and $K^{g}$ is also a complement of $\mathbf{F}(G)$ in $N$. Thus, there is an $n \in N$ such that $K^{g}=K^{n}$ by the second part of the Schur-Zassenhaus theorem. So $g=m_{1} n$ for some $m_{1} \in M$. But $N=\Phi(G) U K$, where $U K \leq M$, so $N=(M \cap N) \Phi(G)$. Thus, $n=m_{2} t$ for some $m_{2} \in M, t \in \Phi(G)$. It follows that $g=m_{1} n=m_{1} m_{2} t \in M \Phi(G)$, and the proof is complete.

Proof of Theorem \%. Suppose that we have the result for groups in consideration with a trivial Frattini subgroup. Then for an arbitrary finite $\pi$-solvable group $G$ where $\pi$ is the set of the prime divisors of $\left|\mathbf{F}^{*}(G)\right|$, we see that $G / \Phi(G)$ is $\mu$-solvable where $\mu$ is the set of the prime divisors of $\left|\mathbf{F}^{*}(G / \Phi(G))\right|$, a subset of $\pi$ by Lemma 16. Therefore

$$
k(G / \Phi(G)) \leq|G / \Phi(G)|_{\mu} \leq|G / \Phi(G)|_{\pi}=|G|_{\pi} /|\Phi(G)|,
$$

since every prime divisor of $|\Phi(G)|$ is in $\pi$. Using Nagao [23], we then deduce that

$$
k(G) \leq k(\Phi(G)) k(G / \Phi(G)) \leq|\Phi(G)| \cdot\left(|G|_{\pi} /|\Phi(G)|\right)=|G|_{\pi},
$$

as wanted. Thus we may assume that $\Phi(G)=1$.

Notice that the hypothesis on $G$ implies that $\mathbf{F}^{*}(G)=\mathbf{F}(G)$ and thus $\mathbf{F}(G)$ is a direct product of elementary abelian groups and $X:=G / \mathbf{F}(G)$ acts faithfully on $V:=\mathbf{F}(G)$. Moreover by [13, III. 4.4], we may also assume that $G$ is the split extension $X V$ of a subgroup $X$ of $G$ and $V$.

We now proceed by induction on the size $n$ of the set $\pi$.

Let $n=1$. Then $V$ is an elementary abelian $p$-group for some prime $p$ and $X$ acts faithfully on $V$. Thus by Lemma 15 we have $k(G) \leq|G|_{p}$, as desired.

So suppose that $n>1$ and that the theorem is true for $n-1$. Let $V=W \oplus U$ where $W$ is the maximal elementary abelian $p$-subgroup of $V$ for some prime divisor $p$ of $V$.

Put $Y:=X U \cong G / W$ so that $G=Y W$. Put $N:=\mathbf{C}_{Y}(W)$. Then $N$ contains $U$ as a normal subgroup and $N$ is normal in $G$. In particular, $\mathbf{F}^{*}(N)=\mathbf{F}(N)$ (since a component in $N$ is also a component in $G$ ). Now notice that $N / U$ acts faithfully on $U$. From this it follows that the sets of the prime divisors of $|\mathbf{F}(N)|$ and $|U|$ are the same and equal to $\mu:=\pi \backslash\{p\}$. As it is clear that $N$ is $\mu$-solvable, the induction hypothesis implies that $k(N) \leq|N|_{\mu}$, which in turn implies that $k(N) \leq|N|_{\pi}$.

Now $G / N \cong(Y / N) W$ and $Y / N$ acts faithfully on $W$. It follows that $|\mathbf{F}(G / N)|$ has exactly one prime divisor, which is $p$. As $G$ is $\pi$-solvable, the quotient $G / N$ is $p$ solvable. Hence by Lemma 15, we have $k(G / N) \leq|G / N|_{p}$. Thus $k(G / N) \leq|G / N|_{\pi}$.

Using Nagao [23] again, we deduce that

$$
k(G) \leq k(N) k(G / N) \leq|N|_{\pi} \cdot|G / N|_{\pi}=|G|_{\pi} .
$$

This completes the proof of the first part of the theorem. The second part follows readily as

$$
|G: H|=|G| /|G|_{\pi} \leq|G| / k(G) \leq b(G)^{2}
$$


For the last part, we just note that if $G$ is solvable then the set of the prime divisors of $\left|\mathbf{F}^{*}(G)\right|$ is precisely that of $|\mathbf{F}(G)|$.

Remark. Notice that the inequality $k(G) \leq|\mathbf{F}(G)|$ implies Gluck's conjecture for the solvable group $G$. However this inequality does not always hold by considering $G=A G L(2,3)$, as kindly pointed out to us by Robinson [27].

As mentioned in the introduction, Theorem 4 is a consequence of results on the so-called commuting probability of finite groups. The commuting probability of a finite group $G$, denoted by $\operatorname{cp}(G)$, is the probability that a randomly chosen pair of elements of $G$ commute. That is,

$$
\operatorname{cp}(G):=\frac{1}{|G|^{2}}|\{(x, y) \in G \times G \mid x y=y x\}| .
$$

It is well-known that $\operatorname{cp}(G)=k(G) /|G|$ where $k(G)$ denotes the number of conjugacy classes in $G$.

Using the classification of finite simple groups and early work of R. Knörr [18] on the coprime $k(G V)$-problem, Guralnick and Robinson [11] obtained a quite strong bound for the commuting probability, namely $\operatorname{cp}(G) \leq|G: \mathbf{F}(G)|^{-1 / 2}$.

Proof of Theorem 4. The result [11, Theorem 10] implies that

$$
\frac{k(G)}{|G|} \leq|G: \mathbf{F}(G)|^{-1 / 2}
$$

which in turn implies that

$$
|G: \mathbf{F}(G)|^{1 / 2} \leq \frac{|G|}{k(G)} .
$$

Since the right-hand side of the previous inequality is no greater than $b(G)^{2}$, we deduce that

$$
|G: \mathbf{F}(G)|^{1 / 2} \leq b(G)^{2}
$$

and the theorem follows.

Remark. Since the proof of the inequality $\operatorname{cp}(G) \leq|G: \mathbf{F}(G)|^{-1 / 2}$ by Guralnick and Robinson depends on the classification of finite simple groups, the above proof of Theorem 4 depends on the classification as well.

Proof of Theorem 8. Using the result [16, Theorem D] of Isaacs and Passman, we have that the nilpotent group $\mathbf{F}(G)$ has a subnormal abelian subgroup, say $A$, of index at most $b(\mathbf{F}(G))^{4}$. Combining this with Theorem 4, we have

$$
|G: A|=|G: \mathbf{F}(G)| \cdot|\mathbf{F}(G): A| \leq b(G)^{4} b(\mathbf{F}(G))^{4} \leq b(G)^{8},
$$

which proves the first part of the theorem. 
The result [20, Theorem 3] of Liebeck and Pyber is that every finite group $G$ contains a solvable subgroup $S$ with $k(G) \leq|S|$. This implies that

$$
|G: S| \leq|G| / k(G) \leq b(G)^{2}
$$

and we have proved the second part of the theorem.

We remark here that it is asked in [20, page 539] whether in fact any finite group $G$ contains a nilpotent subgroup $N$ with $k(G) \leq|N|$. It would be interesting if one could answer the following weaker question, which seems nontrivial to us.

Question 17. Is it true that every finite group $G$ has a nilpotent subgroup of index at most $b(G)^{2}$ ?

\section{ACKNOWLEDGEMENT}

The authors are grateful to David Gluck for his helpful comments and suggestions

on an earlier version of the manuscript. H.N. would like to dedicate this paper to Professor Nguyễn H. V. Hưng for many years of friendship, help, and encouragement.

\section{REFERENCES}

[1] J. H. Conway, R. T. Curtis, S. P. Norton, R. A. Parker, and R. A. Wilson, Atlas of Finite Groups, Clarendon Press, Oxford, 1985.

[2] S. Dolfi, Large orbits in coprime actions of solvable groups. Trans. Amer. Math. Soc. 360 (2008), 135-152.

[3] S. Dolfi and E. Jabara, Large character degrees of solvable groups with abelian Sylow 2subgroups, J. Algebra 313 (2007), 687-694.

[4] A. Espuelas, Large character degrees of groups of odd order, Illinois J. Math. 35 (1991), 499505.

[5] W. Feit, Extending Steinberg characters, Linear algebraic groups and their representations, Contemp. Math. 153 (1993), 1-9.

[6] J. S. Frame, G. B. Robinson, and R. M. Thrall, The hook graphs of the symmetric group, Canad. J. Math. 6 (1954), 316-324.

[7] The GAP Group, GAP - Groups, Algorithms, and Programming, Version 4.6.3, 2013. http://www.gap-system.org

[8] D. Gluck, The largest irreducible character degree of a finite group, Canad. J. Math. 37 (1985), 442-451.

[9] D. Gluck, K. Magaard, U. Riese, and P. Schmid, The solution of the $k(G V)$-problem, J. Algebra 279 (2004), 694-719.

[10] D. Gorenstein, R. Lyons, and R. Solomon, The classifications of the finite simple groups, Math. Surveys Monogr., No. 3, Amer. Math. Soc., Providence, 1998.

[11] R. M. Guralnick and G. R. Robinson, On the commuting probability in finite groups, J. Algebra 300 (2006), 509-528.

[12] Z. Halasi and A. Maróti, The minimal base size of a $p$-solvable linear group, submitted.

[13] B. Huppert, Endliche Gruppen. I. Die Grundlehren der Mathematischen Wissenschaften, Band 134 Springer-Verlag, Berlin-New York, 1967.

[14] I. M. Isaacs, Character theory of finite groups, Dover Publications, New York, 1994. 
[15] I. M. Isaacs, Finite group theory, Graduate Studies in Mathematics, 92. American Mathematical Society, Providence, RI, 2008.

[16] I. M. Isaacs and D. S. Passman, A characterization of groups in terms of the degrees of their characters, Pacific J. Math. 15 (1965), 877-903.

[17] G. D. James, The representation theory of the symmetric groups, Lecture Notes in Mathematics, Springer - Berlin, 1978.

[18] R. Knörr, On the number of characters in a $p$-block of a p-solvable group, Illinois J. Math 28 (1984), 181-210.

[19] M. Larsen, G. Malle, and P. H. Tiep, The largest irreducible representations of simple groups, Proc. Lond. Math. Soc. 106 (2013), 65-96.

[20] M. W. Liebeck and L. Pyber, Upper bounds for the number of conjugacy classes of a finite group. J. Algebra 198 (1997), 538-562.

[21] S. Mattarei, On character tables of wreath products, J. Algebra 175 (1995), 157-178.

[22] A. Moretó and T. R. Wolf, Orbit sizes, character degrees and Sylow subgroups Adv. Math. 184 (2004), 18-36.

[23] H. Nagao, On a conjecture of Brauer for $p$-solvable groups, J. Math. Osaka City Univ. 13 (1962), 35-38.

[24] P.P. Pálfy, A polynomial bound for the orders of primitive solvable groups, J. Algebra 77 (1982), 127-137.

[25] L. Pyber, Personal communication.

[26] G. R. Robinson, Nilpotent injectors and conjugacy classes in solvable groups, J. Aust. Math. Soc. 71 (2001), 349-352.

[27] G. R. Robinson, Personal communication.

[28] P. Schmid, The solution of the $k(G V)$ problem, ICP Advanced Texts in Mathematics 4, Imperial College Press, London, 2007.

[29] Á. Seress, The minimal base size of primitive solvable permutation groups. J. London Math. Soc. 53 (1996) 243-255.

[30] T. R. Wolf, Solvable and nilpotent subgroups of GL $\left(n, q^{m}\right)$, Canad. J. Math. 34 (1982), 10971111.

[31] Y. Yang, Large character degrees of solvable 3'-groups, Proc. Amer. Math. Soc. 139 (2011), 3171-3173.

Department of Mathematics, The University of Akron, Akron, Ohio 44325, USA

E-mail address: cossey@uakron.edu

Department of Algebra and Number Theory, Institute of Mathematics, University of Debrecen, 4010, Debrecen, Pf. 12, Hungary

E-mail address: halasi.zoltan@renyi.mta.hu

Fachbereich Mathematik, Technische Universität Kaiserslautern, Postfach 3049, 67653 Kaiserslautern, Germany and Alfréd Rényi Institute of Mathematics, ReálTANODA UtCA 13-15, H-1053, Budapest, Hungary

E-mail address: maroti@mathematik.uni-kl.de and maroti.attila@renyi.mta.hu

Department of Mathematics, The University of Akron, Akron, Ohio 44325, USA

E-mail address: hungnguyen@uakron.edu 